\title{
Once daily dosing of aminoglycoside: one step forward
}

\author{
J.-C. Pechère", W. A. Craigh and F. Meunieret \\ 'Department of Microbiology, Centre Médical Universitaire, 9 avenue de Champel, \\ 1211 Geneva 4, Switzerland; ' William S. Middelton Memorial Veterans Hospital, 2500 \\ Terrace, Madison, WI 53705, USA; 'Infectious Diseases Unit and Microbiology \\ Laboratory Institut Jules Bordet, 1 rue Heger-Bordet 1000-Brussels, Belgium
}

Aminoglycosides continue to be widely used for treating severe infections (EORTC, 1987; Barrière, 1988), despite their limited antibacterial spectrum, their potential for toxicity and the availability of many other potent antibiotics. Rapid bactericidal activity against susceptible Gram-negative bacilli, and synergism with $\beta$-lactam compounds, further justifies the continuing popularity of the aminoglycosides. Recent experimental data have suggested that increasing dosage intervals while maintaining the usual daily dose would ensure maximum efficacy and limit the development of nephrotoxicity. However, before endorsing these new schedules, several issues deserve careful consideration.

With regard to the pharmacodynamics there is strong support for these new regimens. Various in-vitro and animal models have shown that the higher the concentration of aminoglycoside in these models, the more effective bacterial killing (Flueckiger, Feller \& Gerber, 1986; Vogelman \& Craig, 1986; Dudley \& Zinner, 1991). This concentrationdependent activity favours the concept of increased dosing intervals whereby higher intermittent doses produce higher peak levels and improved bacterial killing. Aminoglycosides also produce significant, concentration dependent, post-antibiotic effects (PAE) against both Gram-positive and Gram-negative organisms (Craig \& Vogelman, 1987). In vivo the PAE is able to maintain inhibition of bacterial growth for several hours when concentrations fall below the MIC. In mice with impaired renal function, aminoglycoside pharmacokinetics have been shown to mimic those in man and ensure antimicrobial activity for approximately $24 \mathrm{~h}$ owing to the PAE. As a consequence, the efficacy of the once daily dosing of amikacin was virtually identical to that observed after administration of the same daily amount of drug given in two or four divided doses (Craig, Redington \& Ebert, 1991). In addition, it is interesting to note that synergistic PAEs have been observed with various combinations of $\beta$-lactams and amikacin (Isaksson et al., 1991).

With respect to pharmacokinetics, when comparing the once-daily and twice-daily regimens of amikacin, most parameters follow an almost linear correlation. With the former schedule, a small reduction of the volume of distribution over time has been shown (Van der Auwera, 1991), causing a modest increase in the peak levels of amikacin during therapy (Viscoli et al., 1991); clinical improvement of the patient may well account for this trend. With both the conventional and new regimens, the volume

"Present address: EORTC Central Office, avenue E. Mounier 83, 1200 Brussels, Belgium. 
of distribution for amikacin shows enormous variation among the populations of patients requiring aminoglycoside therapy for severe infections. Although it seems advisable to monitor amikacin blood levels the peak and trough levels which should be achieved for optimal efficacy and minimal toxicity for the once daily regimen remain to be determined. Pending further clinical evaluation it seems reasonable to avoid trough levels higher than $5 \mathrm{mg} / \mathrm{l}$ (for amikacin) or $2 \mathrm{mg} / \mathrm{l}$ (for other aminoglycosides). Animal models have demonstrated that renal uptake of gentamicin, netilmicin and amikacin, but not that of tobramycin, do not correlate linearly with serum concentrations (Guiliano et al., 1986; Verpooten et al., 1989; De Broe, Verbist \& Verpooten, 1991). Relatively less of the former three aminoglycosides accumulate in the kidney with increases in serum concentrations. It is likely that this observation may explain the lesser nephrotoxicity observed with once-daily regimens, compared to divided daily dosings, as demonstrated in various animal models (Frame et al., 1977; Reiner, Bloxham \& Thompson, 1978; Powell et al., 1983).

Toxicity is an important issue in the debate over once daily dosing. In patients treated with netilmicin, the once-daily regimen delayed the occurrence of nephrotoxicity in comparison with multiple daily dosing (ter Braak et al., 1990). Of the five studies dealing with more than 700 paediatric and adult patients, using the conventional regimen as control, less nephrotoxicity was observed with the once-daily dosing regimen in one study (P. E. Marik, personal communication), a second study supported the same trend (using phospholipiduria as the criterion; Tulkens, 1991), while the three other studies failed to indicate a significant difference (Giamarellou et al., 1991; Maller et al., 1991; S. Lauwers, personal communication). However, in considering aminoglycoside toxicity ototoxicity is also extremely important, since severe irreversible damage can occur, including deafness. Comparative studies are extremely difficult to perform and interpret because baseline auditory function is rarely defined in the severely ill patient requiring aminoglycoside therapy. Furthermore the mechanisms of aminoglycoside ototoxicity remain unclear; aminoglycoside induced inner ear defects do not seem to be associated with drug accumulation as in the case of nephrotoxicity (Tran Ba Huy, Bernard \& Schacht, 1981). However, in a population of young females suffering from pelvic inflammatory disease and without other serious underlying disease, surveillance by high tone audiometry showed a significant safety advantage with the once-daily regimen compared to the divided daily schedules of netilmicin (Tulkens, 1991).

Clinical evaluation of dosing schedules of aminoglycosides with respect to efficacy is difficult largely because these antibiotics are generally prescribed in combination with other agents. Four controlled, randomized, open studies are presented in this issue of the Journal. These patients had various infectious conditions mainly caused by Gram-negative bacilli, and were most often treated with amikacin plus a $\beta$-lactam. One study favoured once daily dosing (Giamarellou et al., 1991), while the others indicated similar efficacy of the two regimens (Maller et al., 1991; Tulkens, 1991; S. Lauwers, personal communication). Other studies, conducted with netilmicin also showed similar efficacy (Fan et al., 1988; Hollender et al., 1989). In patients with neutropenia and various immunodeficiencies, studies published to date are inadequate to assess the efficacy of the once-daily dosing regimen. A large, randomized, controlled study comparing amikacin and ceftriaxone (once daily) with amikacin and ceftazidime (divided dosing) conducted by the European Organization for Research and Treatment of Cancer (EORTC) International Antimicrobial Therapy Cooperative group is awaited with interest. 
In conclusion in non-neutropenic patients with proven or suspected Gram-negative infections for which an aminoglycoside seems appropriate, once daily dosing deserves serious consideration; studies suggest it to be at least as efficacious and as safe as conventional dosing regimens. The new regimen has the obvious advantage of greater convenience, more acceptable to patients, and economic advantages. With regard to neutropenic patients, the results of on-going studies should be awaited before firm conclusions can be made.

\section{References}

Barrière, S. L. (1988). Aminoglycoside: a reassessment of their therapeutic role. Clinical Pharmacology 7, 385-90.

Craig, W. A., Redington, J. \& Ebert, S. C. (1991). Pharmacodynamics of amikacin in vitro and in mouse thigh and lung infections. Journal of Antimicrobial Chemotherapy 27, Suppl. C, $29-40$.

Craig, W. A. \& Vogelman, B. S. (1987). The postantibiotic effect. Annals of Internal Medicine $106,900-2$.

De Broe, M. E., Verbist, L. \& Verpooten, G. A. (1991). Influence of dosage schedule on renal cortical accumulation of amikacin and tobramycin in man. Journal of Antimicrobial Chemotherapy 27, Suppl. C, 41-47.

Dudley, M. N. \& Zinner, S. H. (1991). Single daily dosing of amikacin in an in-vitro model. Journal of Antimicrobial Chemotherapy 27, Suppl. C, $15-19$.

EORTC International Antimicrobial Therapy Cooperative Group (1987). Ceftazidime combined with a short or long course of amikacin for empirical therapy of Gram-negative bacteremia in cancer patients with granulocytopenia. New England Journal of Medicine 317, 1692-8.

Fan, S. T., Lan, W. Y., Teoh-Chan, C. H., Lan, K. F. \& Mauracher, E. M. (1988). Once daily administration of netilmicin compared with thrice daily, both in combination with metronidazole, in gangrenous and perforated appendicitis. Journal of Antimicrobial Chemotherapy 22, 69-74.

Flueckiger, U., Feller, C. \& Gerber, A. U. (1986). Dose-response curves in a man-adapted model: a novel view of comparative in vivo assessment of antibiotics. In Program and Abstracts of the Twenty-Sixth Interscience Conference on Antimicrobial Agents and Chemotherapy, New Orleans, 1986. Abstract 577, p. 202. American Society for Microbiology, Washington, DC.

Frame, P. T., Phair, J. P., Watanakunakorn, C. \& Bannister, T. W. P. (1977). Pharmacologic factors associated with gentamicin nephrotoxicity in rabbits. Journal of Infectious Diseases $135,925-6$.

Giamarellou, H., Yiallouros, K., Petrikkos, G., Moschovakis, E., Vavouraki, E., Voutsinas, D. et al. (1991). Comparative kinetics and efficacy of amikacin administered once or twice daily in the treatment of systemic Gram-negative infections. Journal of Antimicrobial Chemotherapy 27, Suppl. C, 73-79.

Guiliano, R. A., Verpooten, G. A., Verbist, L., Wedeen, R. P. \& De Broe, M. E. (1986). In vivo uptake kinetics of aminoglycosides in the kidney cortex of rats. Journal of Pharmacology and Experimental Therapeutics 236, 470-5.

Hollender, L. F., Bahnini, J., De Manzini, N., Lau, W. Y., Fan S. T., Hermansyur, K. et al. (1989). A multicentric study of netilmicin once daily versus twice daily in patients with appendicitis and other intra-abdominal infections. Journal of Antimicrobial Chemotherapy 23, 773-83.

Isaksson, B., Hanberger, H., Maller, R., Nilsson, L. E. \& Nilsson, M. (1991). Synergistic postantibiotic effect of amikacin and $\beta$-lactam antibiotics on Enterococcus faecalis. Journal of Antimicrobial Chemotherapy 27, Suppl. C, 9-14.

Maller, R., Ahrne, H., Eilard, T., Erikkson, I., Lausen, I. and the Scandinavian Amikacin Once Daily Study Group. (1991). Efficacy and safety of amikacin in systemic infections when given as a single daily dose or in two divided doses. Journal of Antimicrobial Chemotherapy 27, Suppl. C, 121-128.

Powell S. H., Thompson, W. L., Luthe, M. A. S., Stern, R. C., Grossniklaus, D. A., Bloxham, D. D. et al. (1983). Once-daily vs continuous aminoglycoside dosing: efficacy and toxicity in 
animal and clinical studies of gentamicin, netilmicin, and tobramycin. Journal of Infectious Diseases 147, 918-32.

Reiner, N. E., Bloxham, D. D. \& Thompson, W. L. (1978). Nephroxicity of gentamicin and tobramycin given once daily or continuously in dogs. Journal of Antimicrobial Chemotherapy 4, Suppl. A, 85-101.

ter Braak, E., De Vries, P. J., Bouter, K. P., Van der Vegt, S. G., Dorrestein, G. C., Nortier, J. W. et al. (1990). One-daily dosing regimen for aminoglycoside plus $\beta$-lactam combination therapy of ceftriaxone. American Journal Medicine 89, 58-66.

Tran Ba Huy, P., Bernard, P. \& Schacht, J. (1986). Kinetics of gentamicin uptake and release in the rat. Comparison of inner ear tissues and fluids with other organs. Journal of Clinical Investigation 77, 1492-500.

Tulkens, P. M. (1991). Pharmacokinetic and toxicological evaluation of a once-daily regimen versus conventional schedules of netilmicin and amikacin. Journal of Antimicrobial Chemotherapy 27, Suppl. C, 49-61.

Van der Auwera, P. (1991). Pharmacokinetic evaluation of single daily dose amikacin. Journal of Antimicrobial Chemotherapy 27, Suppl. C, 63-71.

Verpooten, G. A., Guiliano, R. A., Verbist, L., Eestermans, G. \& De Broe, M. E. (1989). Once-daily dosing decreases renal accumulation of gentamicin and netilmicin. Clinical Pharmacology and Therapeutics 45, 22-7.

Viscoli, C., Dudley, M., Ferrea, G., Boni, L., Castagnola, E., Barretta, M. A. et al. (1991). Serum concentrations and safety of single daily dosing of amikacin in children undergoing bone marrow transplantation. Journal of Antimicrobial Chemotherapy 27, Suppl. C, 113-120.

Vogelman, B. S. \& Craig, W. A. (1986). Kinetics of antimicrobial activity. Journal of Pediatrics $108,835-40$. 